\title{
A NOVEL CONCEPT FOR A MID-FIELD MICROSCOPE
}

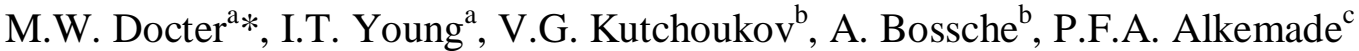 \\ and Y. Garini ${ }^{\mathrm{a}}$ \\ ${ }^{a}$ Imaging Science and Technology, ${ }^{b}$ Micro-Electronics and ${ }^{c}$ Nanoscience departments \\ Delft University of Technology, Faculty of Applied Sciences, \\ Lorentzweg 1, Delft 2628 CJ, The Netherlands
}

* M.W.Docter@tnw.tudelft.nl; phone: +31-15-278-1303, fax: +31-15-278-6740

\begin{abstract}
When light passes through a hole smaller than the wavelength of the light, the transmission is very low and the light is diffracted. This however changes if holes are arranged in a periodic array on metal. In that case the light couples to surface plasmons; this results in enhanced transmission, spectral selection and a small angular diffraction.

We develop a novel microscopic method based on a periodic hole-array, which will be used as a multiple-apertures near-field source for illuminating a biological sample while the light is collected in far-field. The measurement speed is high, due to the use of an array instead of a single source. The main advantage of this microscope originates from the low diffraction of light through a relatively thick sample with enhanced transmission. It results in the ability to measure the samples interior and 3D reconstruction can be made by semi-confocal techniques. This overcomes the major limitation of near-field methods for which only a shallow layer of the surface $(\sim 20 \mathrm{~nm})$ is detectable.

For our measurements we use glass coated devices. The holes are processed with a focused ion beam. The photonplasmon coupling process is characterized as a function of the wavelength. Our experiments aim on gaining a better understanding of the transmission process. We tested the dependence of the transmitted spectrum on angle of incidence was tested as well as far-field spectral imaging measurements of the transmission in both Koehler and collimated light illumination. The results as well as the description of the microscope that we are constructing are presented.
\end{abstract}

\section{Keywords:}

plasmons, mid-field, nanostructure, hole array, near-field, high resolution, microscopy

\section{INTRODUCTION}

The discovery of the microscope by van Leeuwenhoek [1] and others made it possible to observe human cells. The bio-medical research aiming at understanding the mechanisms of the human body made a huge step forward with this discovery. Ever since more bio-medical knowledge is gained and aids to improve diagnostics, prevent and cure illnesses. At present, a lot of effort is invested in understanding intra-cellular mechanisms on the molecular level, including genomic function and structure. This kind of research requires further development of the present microscopy methods to achieve better spatial resolution and higher sensitivity, to name a few.

While the resolution of the early images was limited by the lens aberrations, the diffraction limit nowadays is more easily reached. This resolution limit was defined by Abbe [2] and is proportional to the wavelength divided by the numerical aperture (NA). Though the diffraction limit is continuously challenged, it was hardly broken. Nevertheless, resolution can be improved by using smaller wavelengths or by using more sophisticated optical setups. A common method is used in a confocal microscope that block the "out of focus" light, originating from features outside the plane of interest. It gives an improvement of only a factor $\sqrt{2}$ [3]. Other methods are 
continuously developing and provide resolution that can potentially increase the spatial resolution in an order of magnitude [4].

Another method is to avoid the usage of optical elements for the illumination and significantly decreasing the source size as done in a near-field scanning optical microscope (NSOM) [5]. This method has two major drawbacks; namely the limited intensity due to internal reflections in the glass fiber and the large angular spread at the aperture due to diffraction. However, if the aperture is held in close proximity to the sample, a high resolution scan of the surface is achieved.

In this paper we propose a microscope which will have near-field illumination like the NSOM, and far-field collection like the confocal microscope. Therefore we termed this microscope the mid-field microscope. Its performance, the illumination intensity and the resolution, will lie in between that of the NSOM and confocal microscope.

The near-field illumination will be done through a periodic nano-structure with sub-wavelength holes. Extraordinary transmission occurs through such nano-array. Due to enhancement the intensity will be higher than for the NSOM. More important, as it is expected that very small diffraction will exist, the narrow-beams that illuminates the sample will provide a much higher penetration depth of few micrometers. The resolution will be better than for the confocal microscope, because sub-wavelength nano-apertures will be used. While total diffraction is expected from classical theory, the periodicity of the hole-array enables to achieve very little diffraction of the transmitted beams.

In this article the origin of the extra-ordinary transmission will be further explained. The concept of the mid-field microscope will be described as well as strong evidences supporting the expected characteristics.

\section{LIGHT TRANSMISSION TROUGH SUB-WAVELENGTH HOLE ARRAY}

Using a smaller source size improves the resolution of the system, but reduces the intensity. Bethe [6] predicted the transmission for light through an aperture which has a sub-wavelength size and found that the transmission intensity depends on $(\mathrm{d} / \lambda)^{4}$ and that the transmitted light is completely diffracted.

The work done by Ebbesen and others [7] showed that the theory of transmission through small holes should be modified. This work revealed extra-ordinary transmission when periodic nano-structures are used, which could be consisting of holes or slits and surrounded by grooves or by other holes/slits. The three most outstanding qualities are enhanced transmission, spectral selectivity and -most importantly for our mid-field microscope -a small angular spread. These qualities can be controlled by redesigning the periodic structure. The thickness, grooves and the material choice have an effect on the intensity $[8,9,10]$. The wavelength dependence of the transmission is influenced by the pitch and the angle of illumination [7,11]; grooves at the exit affect the beam confinement [12].

\section{SURFACE PLASMONS OR DIFFRACTED EVANESCENT WAVES}

The extra-ordinary transmission is caused by coupling between light and surface electron states. Recent articles describe somewhat different mechanisms, mainly due to surface plasmons [7] or diffracted evanescent waves [13]. The two approaches will be further examined. Surface plasmons represent electromagnetic (EM) surface waves. Maxwell's theory predicts that EM surface waves can propagate along a metallic surface with frequencies up to a certain limit.

The existence of surface plasmons follows from Maxwell's equation under the assumption that there are no free charges. The following solution complies with the boundary conditions: 


$$
k_{x}=\frac{\omega}{c} \sqrt{\frac{\varepsilon_{1} \varepsilon_{2}}{\varepsilon_{1}+\varepsilon_{2}}}
$$

Here $k_{x}$ is the wave number parallel to the surface, $\omega$ is the wave number, $c$ the speed of light and $\varepsilon_{1,2}$ are the dielectric constants of the array and surrounding material, they depend on the wavelength.

This equation has only one possible solution for the wave number. The value of the wave number depends on the wavelength and the chosen materials.

When plotting the dispersion curve (wave vector versus wave number) for light and surface plasmons, one observes that they do not intersect [14]. This implies that under normal circumstances light will never couple with surface plasmons. When a periodic structure is used, the periodicity introduces K-vectors that together with the wave vector provided by the light leads to the following definition of wave numbers:

$$
\vec{k}_{x}=\vec{k}_{\text {light }}+i \vec{G}_{x}+j \vec{G}_{y}
$$

In the last equation the wave number of light parallel to the surface depends on the angle of incidence. $G_{x}$ and $G_{y}$ reveal the influence of the periodic structure $\left(G_{x}\right.$ is equal to $2 \pi$ times the neighbor number divided by the lattice constant in $\mathrm{x}$-direction, and the same for $\mathrm{G}_{\mathrm{y}}$ in $\mathrm{y}$-direction).

Combining equation 1 and 2 leads to a more complex dependence of $\omega$ on $\mathrm{k}$. This can be visualized by plotting the dispersion curve, in which for each angle of incidence, the wavelength that couples in with the plasmons is calculated. An example of such a dispersion curve is shown in figure 1. It should be mentioned that this model is only an approximation, for consistency with experimental data a fudge factor is required [15].

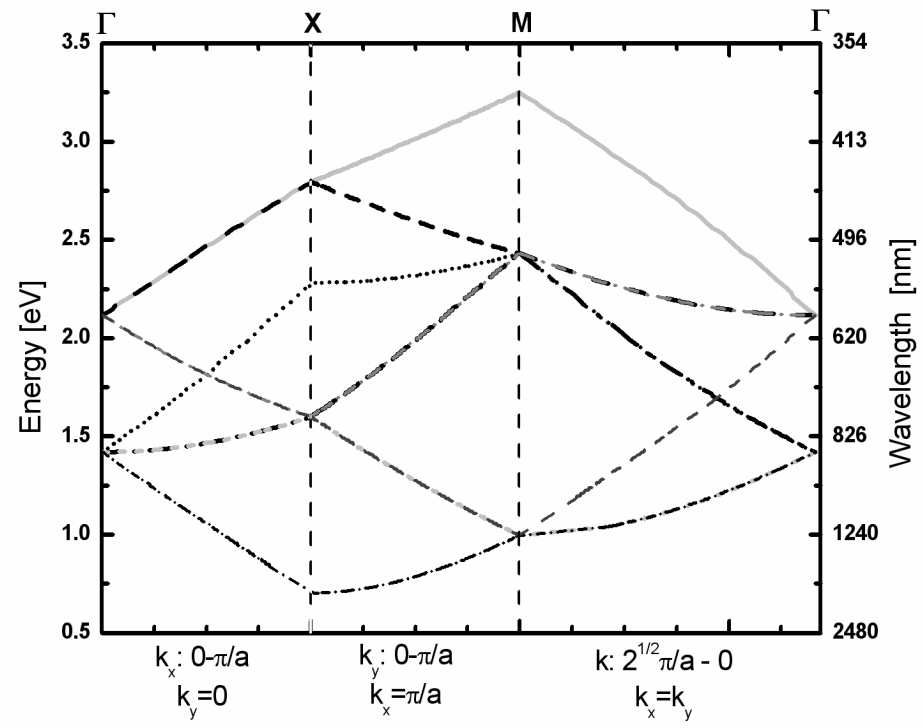

Figure 1. Dispersion curve for gold foil on glass, 25 by 25 holes, a pitch of $600 \mathrm{~nm}$ and a hole diameter of $150 \mathrm{~nm}$.

It is now clear that coupling of light with the surface plasmons is possible, but the reason for transmission enhancement is not explained yet. Surface plasmons exist at both sides of the array. At the front surface, light couples in with the plasmons. These evanescent waves can move around the surface and can penetrate into the holes. They travel evanescently through the holes and can couple back into light at the other side of the array [8].

Some experimental results reveal such a behavior, which cannot be explained by the surface plasmon (SP) theory. For example: the spectral behavior of a metal and non-metal look similar (except for the intensity). Lezec [13] 
introduced another theory which explains the discrepancies in the SP theory with combined diffracted evanescent waves $(\mathrm{CDEW})$.

When light diffracts at a small hole, part of the light is diffracted into radiative modes and the other part into evanescent modes along the surface. These surface modes have $\mathrm{k}_{\mathrm{x}}>\mathrm{k}_{\text {light }}$, after summation of all these modes a running wave propagating with a well-defined wave vector (CDEW) moves in the plane of the surface. The decay of intensity over distance $(x)$ goes with $x^{-1}$ and there is a phase shift between transmitted and surface waves. The transmission qualities are found after the CDEW interfere with the transmitted waves.

For our application we will just use the periodic hole structure; the supporting theory is of second importance. However, some nice features can arise from one of the theories. In the SP theory only one specific mode is considered, while the CDEW theory considers all evanescent modes. Instead of the case of SP, the CDEW does not require a metal, which would broaden our choice of array material. For the CDEW no fudge factor is required when comparing theory and experimental data, while the SP data and theory are not directly comparable.

\section{THE 3D MID-FIELD MICROSCOPE}

The mid-field microscope is a transmission microscope, in which the light transmitted through the array will illuminate a biological sample stained with fluorescent molecules. The fluorescence mode will also allow us a better observation of the three-dimensionality of the object. For transmitted light it is not possible to focus on each plane of interest; the measurements are influenced by the total light path. However, each fluorescent molecule will act like a point source. This makes it possible to focus and detect the fluorescence in that particular plane Focusing each time on a different plane will provide the 3D data.

The setup of the microscope is displayed in figure 2. It can be divided into three parts: i. the illumination, ii. the array and sample and iii. the collection optics. For the illumination we require collimated light of a certain wavelength; the spectral selectivity of the hole-array will further narrow the spectral range that is used for the excitation. The selected wavelength should match the excitation wavelength of the fluorescent molecules. This can be done by adjusting the pitch of the array after choosing the adequate fluorescent molecule. The requirement for collimation originates from the angular dependence of the transmission [7]. Light that illuminates the hole-array in an angle will require a different wavelength so that it can be efficiently transmitted. Having illumination perpendicular to the array causes the intensity to be maximal for perpendicular entrance, from which follows the required collimation.

The close proximity between the array and sample will allow taking full advantage of the confinement of the beam, which is limited to a short distance close to the surface. During the measurement, each plane has to be scanned to ensure the illumination of all the area of the sample. This will be made by a piezoelectric positioner. Scanning different planes is performed by moving the position of the objective relative to the sample.

A single image acquired from this system displays an array of spots, all with a different intensity according to the fluorescent molecules distribution. Even though each spot will be imaged as the point spread function (PSF), this is not a problem as long as the PSF's don't overlap. After scanning in three dimensions, imaging analysis will be done combining all the spot images into a $3 \mathrm{D}$ image.

Some technical difficulties cannot be fully predicted, like the amount that the stationary array and moving sample affect each others motion. These issues will be solved before the microscope works as wanted.

The typical resolution of the microscope will depend on the size of the holes. Our present arrays have holes ranging from 50 up to $150 \mathrm{~nm}$. This resolution will also depend on the distance from the sample to the array, due to the small but not negligible angular spread. We estimate that the microscope will be efficient for a sample depth of few micrometers; the exact number will follow from high-resolution near-field measurements on the beam expansion. We have not conducted these measurements yet, but our far-field measurements provide strong evidence supporting the small angular spread. 


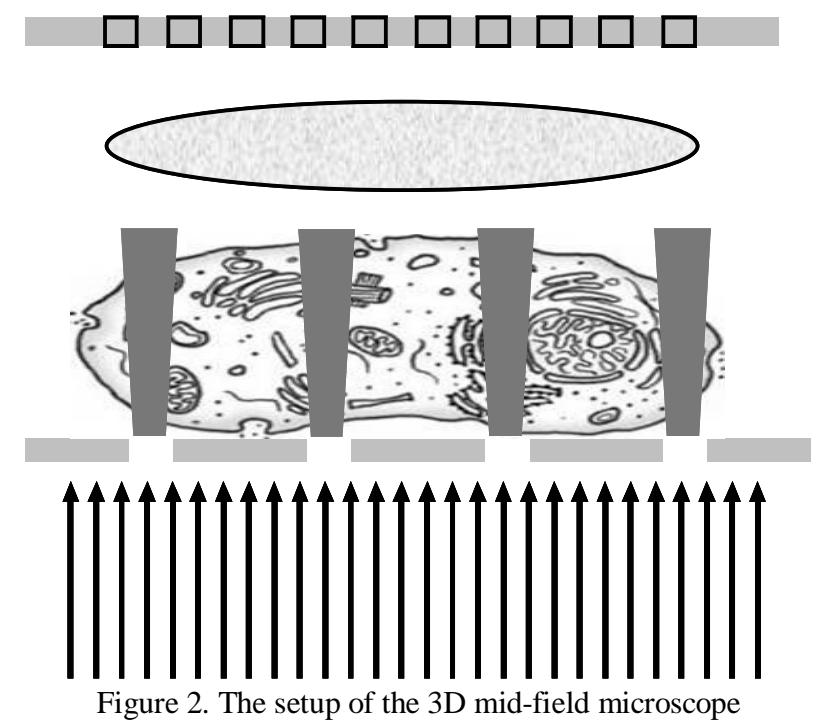

\section{MEASUREMENTS}

All measurements are conducted on the array, in order to gain more knowledge about its transmission behavior. This was done in the setup described in section 4 adapted to an upright optical microscope (Leica DM-RXA), with a controllable stage (smallest move of $0.1 \mu \mathrm{m}$ ). For getting a collimated beam, an extra lens ( $\mathrm{f}=250 \mathrm{~mm}$ ) was added in between the field and condenser lens and the high NA condenser lens was changed for a lower one. The collimation is tested by measuring the beam divergence (an angle of $2^{\circ}$ is achieved).

Data was acquired with a spectral imaging system (SpectraCube, Applied Spectral Imaging) that allows to measure the image with a high spectral resolution, and at the same time to measure the fluorescent spectrum at each pixel (spectral resolution of approximately $10 \mathrm{~nm} @ 550 \mathrm{~nm}$ ). We measured mainly in the spectral range of 400 and 800 $\mathrm{nm}$, where the sensitivity of the system is optimal. The transmitted spectra are corrected by dividing it with the spectrum of the light source (as measured in exactly the same setup without the sample in place). To the best of our knowledge, this is the first time that spectral images of hole-arrays are being measured.

Two kinds of measurements are described. The first was aimed to check whether our hole-arrays are comparable to the ones in literature and the second was done in far-field with different illuminations (Koehler and collimated). The spectral imaging software allows analyzing the data, e.g., by stepping trough the spectral images, such that the intensity image for each wavelength is observed. It also allows observing the image after integrating a selected spectral range (for example 550-650 $\mathrm{nm}$ ).

\subsection{Spectral selectivity of hole-arrays}

We have measured the transmission as a function of the angle of incidence of the illuminated beam. When the angle of incidence changes, so does the wave vector component that is parallel to the surface, and different SP modes are excited. As a result, the transmitted spectrum splits into two peaks that shift with growing angle (figure 3 ).

The sample is made of $25 * 25$ holes (Au on glass) with a $600 \mathrm{~nm}$ pitch and $150 \mathrm{~nm}$ hole diameter. The tilt angle was estimated from the geometry of the mechanism used to tilt the sample. 

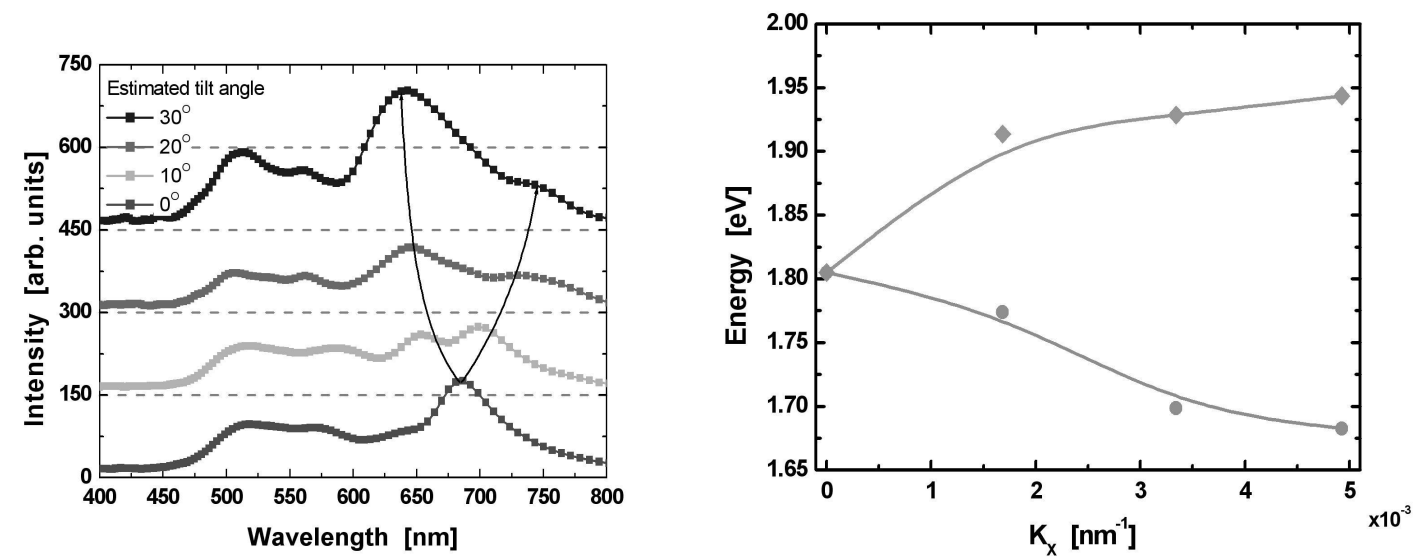

Figure 3. The splitting of peaks. At the left the different spectra for each (estimated) tilt angle are shown, at the right dispersion curve is plotted (the peak position is plotted for different wave numbers).

By calculating the energy $E$ from the wavelength $\lambda$ (equation 3), and the in-plane wave vector $k_{x}$ from both wavelength and angle $\alpha$ (equation 4) where $c$ is the speed of light $\left(3.0 \cdot 10^{8} \mathrm{~ms}^{-1}\right)$ and $h$ is Planck's constant $\left(6.63 \cdot 10^{-34} \mathrm{Js}\right)$, the dispersion that we have measured (figure 3$)$ can be compared with results form other samples. The peak split up in two as expected. The quantitative comparison of the energy $(1.8 \mathrm{eV})$ is in agreement with our calculation (figure 1) when a fudge factor of 1.28 is used. This is in reasonable agreement with other publications described for different hole arrays [15]. The difference is probably due to the fact that the model neglects the actual hole size of the hole arrays and the non-perfection of the dispersion curve.

$$
\begin{aligned}
& E=\frac{h c}{\lambda} \\
& k_{x}=\frac{2 \pi}{\lambda} \sin \alpha
\end{aligned}
$$

\subsection{Measurements with Koehler and collimated illumination}

Collimated illumination will eventually be used within the mid-field microscope. However images under different illuminations can provide important information. Besides collimated illumination we can also use Koehler illumination (figure 4). There are two main differences in illumination, namely the collection of angles and the intensity (high intensity and many incidents angles for Koehler compared to collimated light with only one incident angle and low intensity).

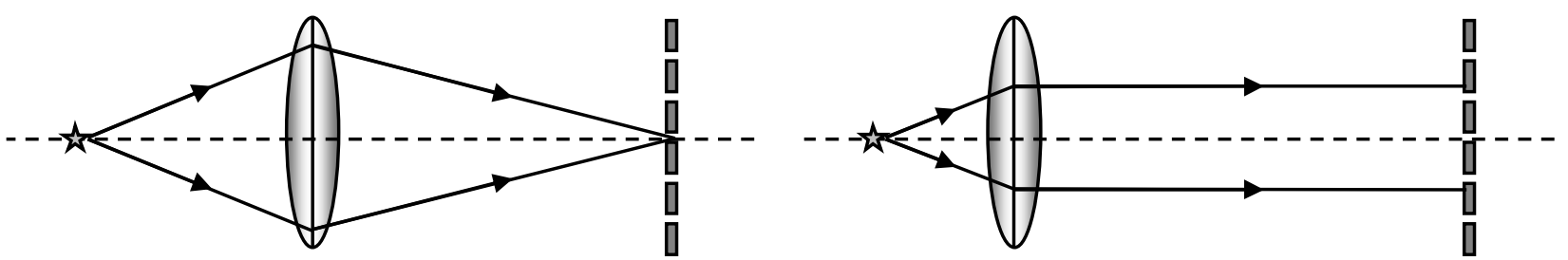

Figure 4. Different illumination: Koehler (left) and collimated (right)

Some wavelengths will be enhanced, others reduced; the extra-ordinary transmission is wavelength dependent. Light of certain wavelength will couple in, the other wavelengths will not. This means that two possible ways of transmission are possible, namely coupled and non-coupled; the later is diffraction (figure 5). In case of coupling, the beam will be collimated for a few micrometers; after that region it is not known. 


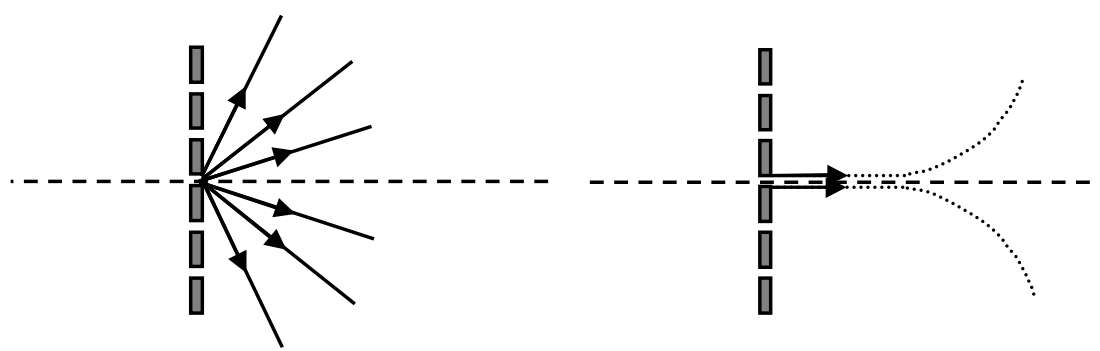

Figure 5. Different transmission: diffracted (left) and coupled (right).

Observation of a sharp image implies that the light coming out of each hole acts like a point-source. The opposite case is such that the light being transmitted from each hole acts like a collimated beam. In such a case, the wave front is not spherical anymore, the light cannot be focused with the microscope and the image will look blurred.

The images were recorded with an infinity-corrected setup, in which it is easy to change the focus plane of the microscope. The quality of the image can be further tested by changing this focus plane. If the holes acts like pointsources, than the image should be blurred as soon as it gets out of focus. In case of confinement in the near-field of the array the wavefront might differ from the one of a point source. If it is spherical a virtual point source can be found at a plane different from the exit surface. For a non-spherical wave front such a virtual source cannot be found.

The reason we treat these extremes is because the transmission through the holes, which later on will be used to illuminate the sample with an array of sources will behave either like a point source, a collimated source or something in between. We expect that in case of no coupling of the light with the surface plasmons the transmitted light will be totally diffracted; total diffraction means that each hole is actually a point source. In case of coupling, it is expected that the angle will be limited (in the extreme case there will be no diffraction at all). It is also expected that the diffraction will depend on the distance from the surface. At short distance (up to few $\mu \mathrm{m}$ ) the field applied by the surface plasmons can be superimposed with the diffracted beams so that the diffraction will be limited. On the other hand, at larger distances from the array, it is still expected that the beam will have a certain extent of diffraction.

Information about the coupling and the behavior of the transmitted light through a hole can be retrieved from the image. If an array is in focus, the source is a point source. If the array is not in focus, a scan through focus by moving scanning along the optical axis with the objective lens might reveal more information about the light path.

The transmission through an Au on glass hole array (250 nm gold with $2 \mathrm{~nm}$ titanium as adhesion layer) is shown in figure 6 . The hole array consists of $25^{*} 25$ holes with a diameter of $150 \mathrm{~nm}$ and pitch of $700 \mathrm{~nm}$. Images are shown for the in-focus position and Koehler illumination. They are shown in different wavelength ranges, namely 450-550 $\mathrm{nm} ; 550-650 \mathrm{~nm}$ and $650-750 \mathrm{~nm}$.

Observing the pictures reveals the following:

1. In all wavelength ranges for Koehler illumination the array is focused. Koehler illumination implies illumination in all possible incident angles within the illumination numerical aperture. Because the illumination light contains also all the possible wavelengths, each different wavelength can be coupled and transmitted at a different angle, and as a result each hole acts like a point source. As mentioned before, a point source is imaged on the CCD and result in a focused image of the hole-array.

2. In the wavelength range $450-550 \mathrm{~nm}$ only the array itself is visible for both kinds of illuminations. At larger wavelength bright spots appear which are located both on top of the array and outside it. These spots do not result from direct transmission since they are not visible in the entire wavelength region. This implies that the light from the spots entered through the array. The precise mechanism is probably due to structuralimperfection in the nanometer-scale which also provides radiative states [16]. Further spectral information is necessary for understanding the origin of the spectrum. 
$700 \mathrm{~nm}$ pitch
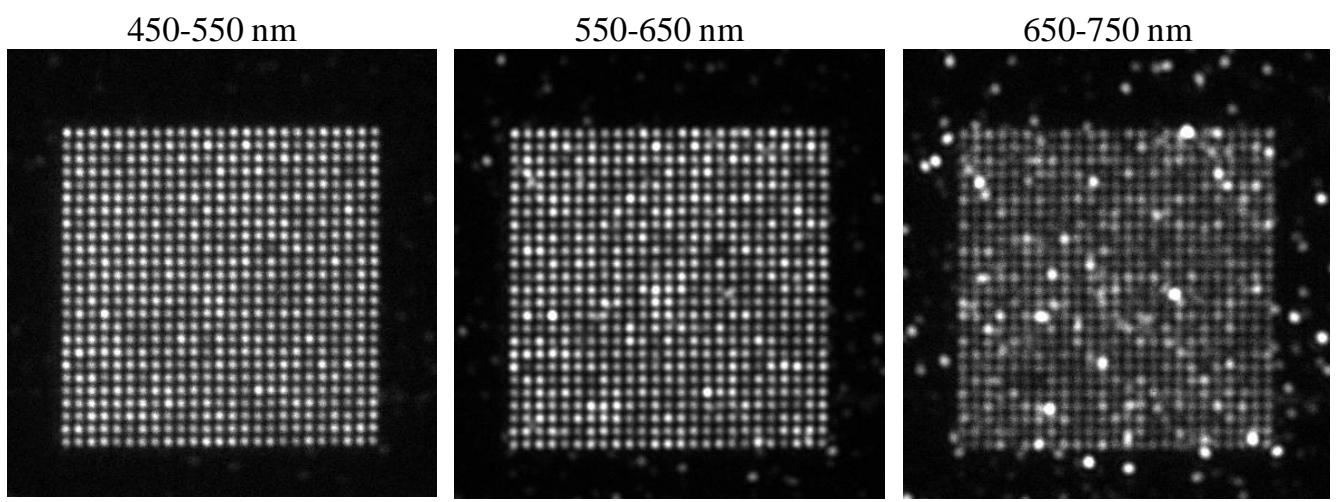

Figure 6. The transmission pictures of the $25 * 25$ hole array, $150 \mathrm{~nm}$ hole diameter and $700 \mathrm{~nm}$ (lower part) pitch

\section{DISCUSSION AND CONCLUSION}

The extra ordinary transmission through a sub-wavelength hole array seems as a promising source for nondiffracted beams. When such an array of nanobeams illuminate a biological sample stained with fluorescent fluorochrome, it can allow to obtain images with a resolution that is better than the diffraction limit. From the resolution of the mid-field microscope (which is better than that of wide-field microscopy) and the depth of the sample that can be measured (which is better than that of a near-field microscope), it would take its position in between the existing near and far-field microscopes. The precise resolution and maximum scan depth are still to be determined by careful measurements of the near-field behavior of the beams. Existing theoretical models (e.g. [17]) are able to predict the near field properties, but to the best of our knowledge no actual measurements have been conducted on transmission through the nano-arrays.

The reported measurements were performed in far-field. Even though the spatial resolution is not as good as can be achieved by near-field, interactions of the scanning tip and the sample in near-field might significantly distort the results. Angular-dependence measurements of the transmitted spectrum showed that the results resemble the theory, though the numerical values can only be fitted by using a fudge factor. This is probably due to the relatively simple model that is used for the calculation and, as an example, does not take into account the hole shape and size.

The spectral images revealed two important issues. First, the individual spots can always be focused for Koehler illumination. This is according to our predictions; future experiments will tell whether there is the same consistency for collimated illumination. Secondly, the results emphasized that spectral-imaging provides necessary information that can not be found by either a single spectrum measurement or an intensity image. From our results it is obvious that part of the intensity (especially in the long-range spectrum) does not originate directly from the array, while it contributes to the spectrum.

So far, our measurements have been conducted with Koehler illumination. More far-field measurements with collimated illumination have to be done and analyzed in order to get a better characterization of the transmission. Our plans also include near-field measurements, which could be compared to the far-field measurements.

We are presently also working on the setup of the 3D midfield microscope. We expect to give a proof of concept of a working $3 \mathrm{D}$ mid-field microscope in the near future.

\section{ACKNOWLEDGEMENTS}

This work was partially supported by the Physics for Technology program of the Foundation for Fundamental Research in Matter (FOM), TNO and the Cyttron Consortium (ICES/KIS Grant BSIK03036). 


\section{REFERENCES}

[1] A. van Leeuwenhoek, Letter to the Royal Society, June 121716

[2] E. Abbe, "Beitrage zur Theorie des Mikroskops und der Mikroskopischen Wahrnehmung", Archiv fur Microskopische Anatomie, 9, 413-420 (1873)

[3] T. Wilson and C. Sheppard, "Theory and practice of scanning optical microscopy", London: Academic Press (1984)

[4] Y. Garini, B.J. Vermolen, I.T. Young, "From micro to nano: recent advances in high-resolution microscopy", Current Opinion in Biotechnology 16 (2005, in press)

[5] E. Betzig, J.K. Trautman, T.D. Harris, J.S. Weiner and R.L. Kostelak, "Breaking the diffraction barrier: optical microscopy on a nanometric scale", Science 251, 1468-1470 (1991)

[6] H.A. Bethe, "Theory of diffraction by small holes", Phys. Rev. 66, 163-182 (1944)

[7] T.W. Ebbesen, H.J. Lezec, H.F Ghaemi, T.Thio and P.A. Wolff, "Extraordinary optical transmission through sub-wavelength hole arrays", Nature 391, 667-669 (1998)

[8] A. Degiron and T.W. Ebbesen, "Analysis of the transmission process through single apertures surrounded by periodic corrugations", Optics express 12, 3694-3700 (2004)

[9] D.E. Grupp, H.J. Lezec and T.W. Ebbesen, "Beyond the Bethe Limit: Tunable Enhanced Light Transmission Through Sub-Wavelength Aperture", Adv. Mat. 11, 860-862 (1999)

[10] D.E. Grupp, H.J. Lezec, T.W. Ebbesen, K.M. Pellerin and T. Thio, "Crucial role of metal surface in enhanced transmission through subwavelength apertures”, Appl. Phys. Lett. 77, 1569-1571

[11] T.Thio, H.F. Ghaemi, H.J. Lezec, P.A. Wolff and T.W. Ebbesen, "Surface-plasmon-enhanced transmission through hole arrays in Cr films", J. Opt. Soc. Am. B. 16, 1743-1748 (1999)

[12] H.J. Lezec, A.Degiron, E. Devaux, R.A. Linke, L. Martin-Moreno, F.J. Garcia-Vidal and T.W. Ebbesen, "Beaming light from a subwavelength Aperture", Science 297, 820-822 (2002)

[13] H.J. Lezec and T. Thio, "Diffracted evanescent wave model for enhanced and suppressed optical transmission through subwavelength hole arrays", Optics express 12, 3629-3651 (2004)

[14] Y. Garini, V. G. Kutchoukov, A. Bossche, P.F.A. Alkemade, M.W. Docter, P.W. Verbeek, L. J. van Vliet and I.T. Young," Toward the development of a Three-Dimensional Mid-Field Microscope", Proceedings of SPIE 5327, 115-122 (2004)

[15] Q.H. Park, D.S. Kim and Ch. Lienau, "Role of surface plasmons in metallic nanoslits", Presentation during the $8^{\text {th }}$ International Conference on Near-field nano-optics \& related techniques (NFO-8, Seoul)

[16] H. Raether, Surface plasmons on smooth and rough surfaces and on gratings, Springer-Verlag 1988

[17] L. Martin-Moreno, F.J. Garcia-Vidal, H.J. Lezec, A. Degiron and T.W. Ebbesen, "Theory of Highly Directional Emission from a Single Subwavelength Aperture Surrounded by Surface Corrugations", Phys. Rev. Lett 90, 167401 (2003) 